\title{
Quadrivalent HPV vaccine efficacy against disease related to vaccine and non-vaccine HPV types in males ${ }^{\text {is }}$
}

\author{
Stephen E. Goldstone ${ }^{\mathrm{a}, *}$, Heiko Jessen ${ }^{\mathrm{b}}$, Joel M. Palefsky ${ }^{\mathrm{c}}$, Anna R. Giuliano $^{\mathrm{d}}$, Edson D.

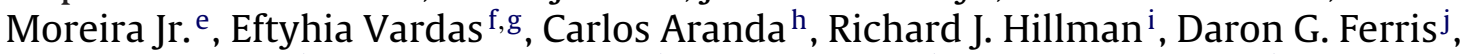 \\ Francois Coutlee ${ }^{\mathrm{k}}$, J. Brooke Marshall ${ }^{1}$, Scott Vuocolo ${ }^{1}$, Richard M. Haupt ${ }^{1}$, Dalya Guris ${ }^{1}$, \\ Elizabeth Garner ${ }^{1}$
}

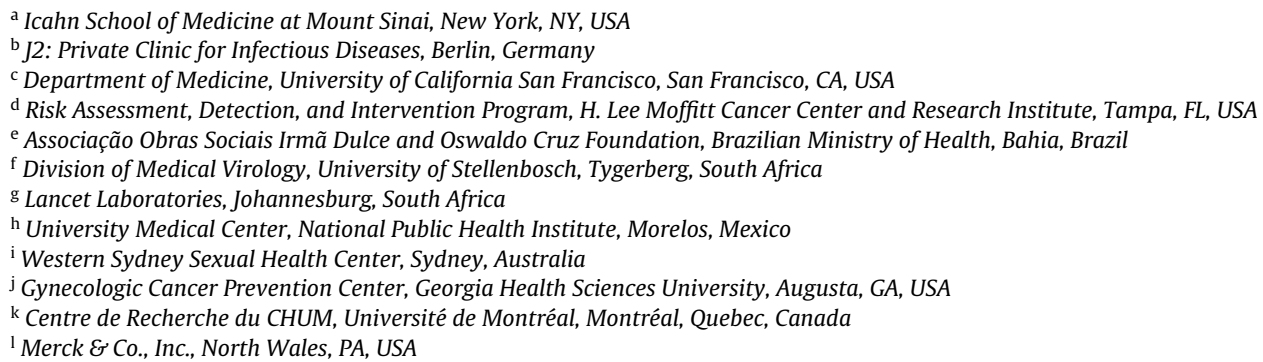

\section{A R T I C L E I N F O}

\section{Article history:}

Received 1 May 2013

Received in revised form 5 June 2013

Accepted 19 June 2013

Available online 2 July 2013

\section{Keywords:}

HPV

Young

Males

Men who have sex with men

\begin{abstract}
A B S T R A C T
A small number of HPV types are related to a majority of HPV-related neoplastic lesions in humans. High-risk types such as HPV 16 and 18 are most often implicated, although other oncogenic and nononcogenic HPV types can cause disease in men. The efficacy of the quadrivalent HPV vaccine (qHPV) against external genital lesions and intra-anal disease related to HPV in men has been demonstrated. This report examines the vaccine's efficacy against disease due to 10 additional non-vaccine HPV types, as well as efficacy regardless of HPV detection. The data presented suggest that vaccinating males against HPV 6, 11, 16 and 18 protects them against most vaccine HPV-type related anogenital disease. However, significant efficacy against disease due to non-vaccine HPV types was not seen. In addition, the data do not provide any evidence that vaccination with qHPV vaccine will increase the likelihood of disease caused by non-vaccine types in the short term.
\end{abstract}

(c) 2013 Elsevier Ltd. All rights reserved.

\section{Background}

Genital infection with the human papillomavirus (HPV) is common in men; the lifetime risk of acquiring an anogenital HPV infection exceeds 50\% [1,2]. Over 40 HPV types (the alpha genus) are capable of infecting the genital epithelium, though not all of these types are oncogenic. Consequences of exposure to these HPV types can include asymptomatic infection, genital warts [3], and a variety of premalignant and malignant lesions of the anogenital epithelium [4,5]. A small number of HPV types are associated with most HPV-related neoplastic disease, with the high-risk types such

\footnotetext{
is $\mathrm{NCT} \# 00090285$.

* Corresponding author at: 420 West 23rd Street, New York, NY 10011, USA.

E-mail address: segmd@prodigy.net (S.E. Goldstone).
}

as HPV 16 and 18 most often implicated in cancers, and HPV 6 and HPV 11 implicated in a majority of genital warts cases [6-8].

Recognized high-risk HPV types within the alpha genus include HPV 16, 18, 31, 33, 35, 39, 45, 51, 52, 56, 58 and 59 [9,10]. All of these HPV types with the exceptions of HPV 51 and HPV 56 are classified in either the A9 or A7 species, of which vaccine HPV types 16 and 18 are respective members. As such, the L1 proteins of HPV 16 and 18 share varying degrees of homology with A9 species members (HPV 31/33/35/52/58) and A7 species members (HPV 39/45/59), respectively. This similarity has led many to question whether vaccines containing antigens to HPV 16 and HPV 18 could generate antibody responses that would protect against related HPV types. While modest efficacy against non-vaccine HPV types has been seen in women aged 16-26 years after receiving the quadrivalent HPV vaccine (qHPV) [11,12], little is known about the efficacy of the vaccine against non-vaccine HPV types in males. 


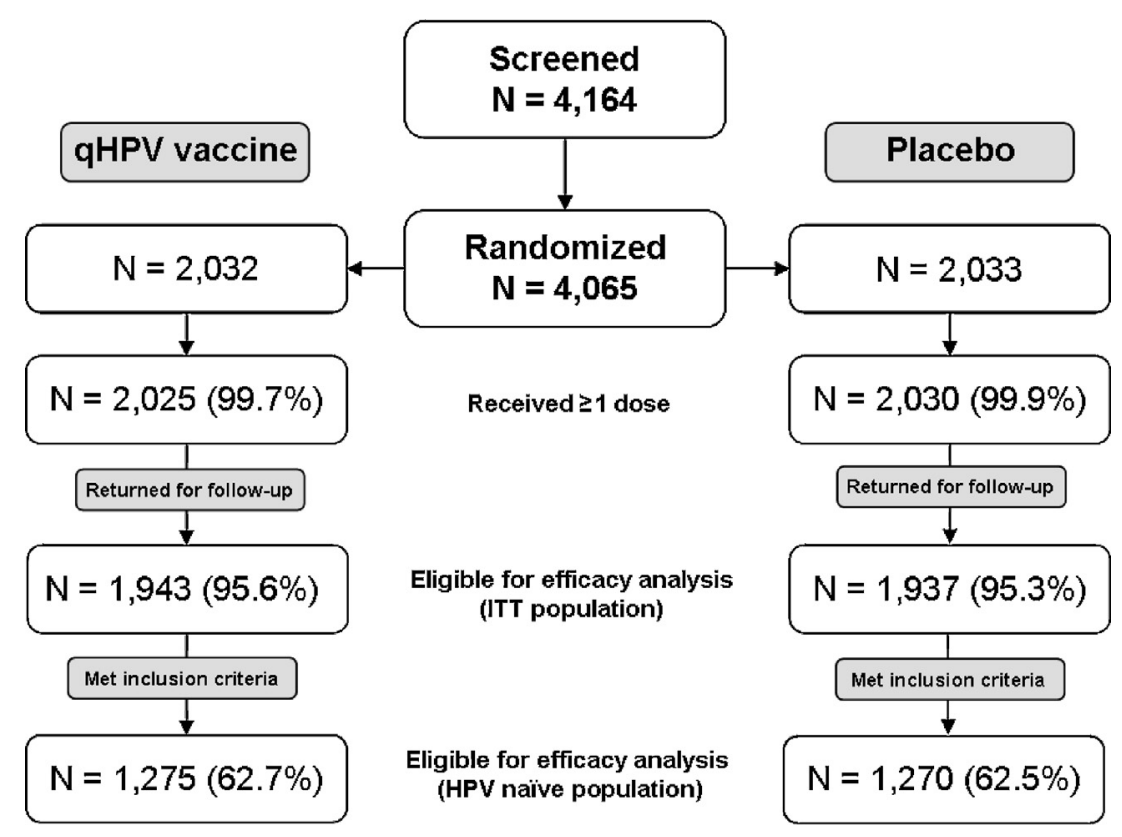

Fig. 1. Accounting of men in the HPV-naïve and intention-to-treat populations.

Here we present data on the efficacy of the qHPV vaccine against external genital lesions (EGL) and anal intraepithelial neoplasia (AIN) related to non-vaccine HPV types in males.

\section{Methods}

\subsection{Study design}

Protocol 020 was designed to evaluate the efficacy of quadrivalent HPV (types 6/11/16/18) L1 virus-like particle vaccine in males (GARDASIL $^{\text {TM }}$, Merck \& Co., Inc., Whitehouse Station, NJ) [13]. The study enrolled 3463 heterosexual males aged $16-24$ years and 602 men who have sex with men (MSM) aged 16-27 years with less than six lifetime sexual partners (Fig. 1). The primary efficacy objective was to demonstrate that, compared with placebo, qHPV vaccine reduced the incidence of HPV 6-, 11-, 16-, or 18related EGL. The secondary efficacy objectives were to demonstrate that, compared with placebo, qHPV vaccine reduced the incidence of HPV 6-, 11-, 16-, or 18-related: (1) persistent infection and (2) DNA detection at any time. The study was not powered to detect efficacy against infection or disease related to individual HPV types.

Participants were enrolled from 71 sites in 18 countries from Africa, Australia, Europe, Latin America and North America. MSM participants were enrolled from 17 sites in Australia, Brazil, Canada, Croatia, Germany, Mexico, Spain and the United States. Males with a history of, or with current clinically detectable HPV-related genital lesions or other sexually transmitted infections were excluded. HPV or cytologic pre-screening was not performed to determine eligibility for enrollment into the study.

\subsection{Study measurements}

All enrolled participants underwent external genital lesion inspection using a magnifying apparatus and sampling for HPV DNA detection at baseline. Participants had serum collected for HIV and syphilis testing at baseline. Serum was also tested for the presence of HPV antibodies as reported previously [13]. A ThinPrep ${ }^{\mathrm{TM}}$ anal cytology specimen was also collected from MSM at baseline and analyzed at a central cytology laboratory. Cytology specimens were evaluated using the Bethesda System-2001, the same classification used for cervical cytology [14].

If a lesion observed at baseline was judged by the investigator to be possibly HPV-related or of unknown etiology, then the participant was excluded from the study. Participants with known immunodeficiency or HIV infection were also excluded. Participants with HIV infection detected after enrollment continued in the study.

Detailed anogenital examinations were scheduled at day 1 and months 7, 12,18,24,30, and 36. EGL judged by the investigator to be possibly, probably, or definitely HPV-related, or any lesion whose etiology was not known were biopsied. Repeated biopsy of recurrent lesions (occurring $<2$ months at same location and appearance) was not performed, to avoid overestimation of incident EGL. All biopsy specimens were processed independently to prevent contamination of HPV DNA and were read in a blinded fashion first for clinical management by pathologists at the central laboratory, then for endpoint adjudication by a panel of pathologists. HPV testing on thin-sections was performed at a central laboratory using multiplex polymerase chain reaction (PCR) $[15,16]$.

Swab samples for HPV testing were collected separately from the penis, scrotum, and perineal/perianal regions, using a nail file/swab system at day 1 and months 7, 12, 18, 24, 30 and 36 [17]. Intra-anal specimens using a Dacron ${ }^{\mathrm{TM}}$ swab were collected from MSM only. All specimens were tested for HPV DNA to identify participants who were infected prior to enrollment and those who acquired new HPV infections during the study. As with the biopsy specimens, each swab sample specimen was evaluated with three different primer-pair sets per HPV type, which amplified a portion of three separate open reading frames.

All swab specimens were tested for the $\beta$-globin gene (positive control), and adequate samples were tested for a panel of 14 HPV types (HPV 6, 11, 16, 18, 31, 33, 35, 39, 45, 51, 52, 56, 58 and 59). Biopsy samples were also tested by PCR for all 14 HPV types. Swab, biopsy (both external and intra-anal), and serum samples were tested at Merck Research Laboratories (Wayne, PA) and Pharmaceutical Product Development (PPD, Wilmington, DE). Detailed procedures have been published previously [18]. 


\subsection{Populations}

Participants were eligible for the HPV-naïve population if they were PCR-negative to all of the 14 tested HPV types and seronegative to HPV types $6,11,16$ and 18 at baseline. In addition, the MSM cohort was required to have a normal anal cytology test at baseline; case counting began after day 1 (Fig. 1 ). While all participants contributed to EGL endpoints (both disease and infection), only the MSM subgroup of 598 participants was eligible for intra-anal endpoints of disease and/or infection.

To be eligible for the intention-to-treat (ITT) population, participants had to receive at least one dose of vaccine or placebo and return for at least one follow-up visit. Case counting for this population began after day 1 .

\section{Results}

Of the 4164 participants screened 4065 were randomized and 4055 received $\geq 1$ dose of $\mathrm{qHPV}$ vaccine or placebo (Fig. 1). Of these participants, 1943 who received qHPV vaccine and 1937 who received placebo returned for follow-up and were eligible for analysis in the ITT population. The HPV naïve population consisted of 1275 participants in the vaccine group and 1270 participants in the placebo group who were seronegative and PCR negative at enrollment to vaccine HPV types 6, 11, 16 and 18, PCR-negative at enrollment to non-vaccine HPV types 31, 33, 35, 39, 45, 51, 52, 56,58 and 59 , who received at least one dose of study material and returned for at least one follow-up visit.

Baseline cohort demographics and HPV sero-prevalence and PCR-prevalence were previously reported [18,19]. Briefly, mean participant age was 20.5 years and the majority of participants were uncircumcised (61.9\%) and Caucasian (35.2\%). Overall 25.2\% of the ITT population was PCR-positive to at least one of 14 tested HPV types (HM 20.9\%, MSM 48.1\%) and 7.6\% were sero-positive to either HPV $6,11,16$ or 18 at baseline (HM 5.0\%, MSM 22.8\%).

Previous reports have demonstrated the efficacy of the qHPV vaccine against HPV 6/11/16/18-related genital lesions in men $[13,20]$. The current report extends the analysis to a more conservative group of men naïve to $14 \mathrm{HPV}$ types at the time of enrollment and also includes lesions in which no HPV type was detected. In this group of men, the efficacy of qHPV vaccine against only the 10 non-vaccine HPV types was $67.1 \%$ (95\% CI: -83.9, 96.8) with 2 vaccine cases and 6 placebo cases (not statistically significant; Table 1). When all cases of EGL (cases related to any tested HPV type and cases where no HPV was identified) were considered in the HPV naïve population a total of 7 cases in the vaccine group and 37 in the placebo group were identified, resulting in an efficacy of 81.5\% (95\% CI: 58.0, 93.0) (Table 1). EGLs due to any one of 10 individual non-vaccine types are detailed in Table 1 ; no statistically significant type-specific efficacy was demonstrated.

The efficacy in the ITT population against EGLs related to the 10 non-vaccine HPV types was 50.3\% (95\% CI: -16.5, 80.3) with 9 cases in the vaccine group and 18 cases in the placebo group (not statistically significant; Table 1). When all cases of EGL were considered in the ITT population (cases related to any tested HPV type and cases where no HPV was identified), there were 38 cases in the qHPV group and 92 cases in the placebo group, resulting in a vaccine efficacy of $59.3 \%$ (95\% CI: 40.0, 72.9) (Table 1).

The rate of any EGL in the ITT placebo group was 1.9 per 100 person years at risk and the rate of EGL related to vaccine-related HPV types (6/11/16/18) in the ITT placebo group was 1.6 per 100 person years at risk (Table 1 ). This compares to a rate of non-vaccine type related (HPV 31/33/35/39/45/51/52/56/58/59) EGL in the ITT placebo group of 0.4 per 100 person years at risk. Table 2 reports the efficacy for both the HPV-naïve and ITT populations against
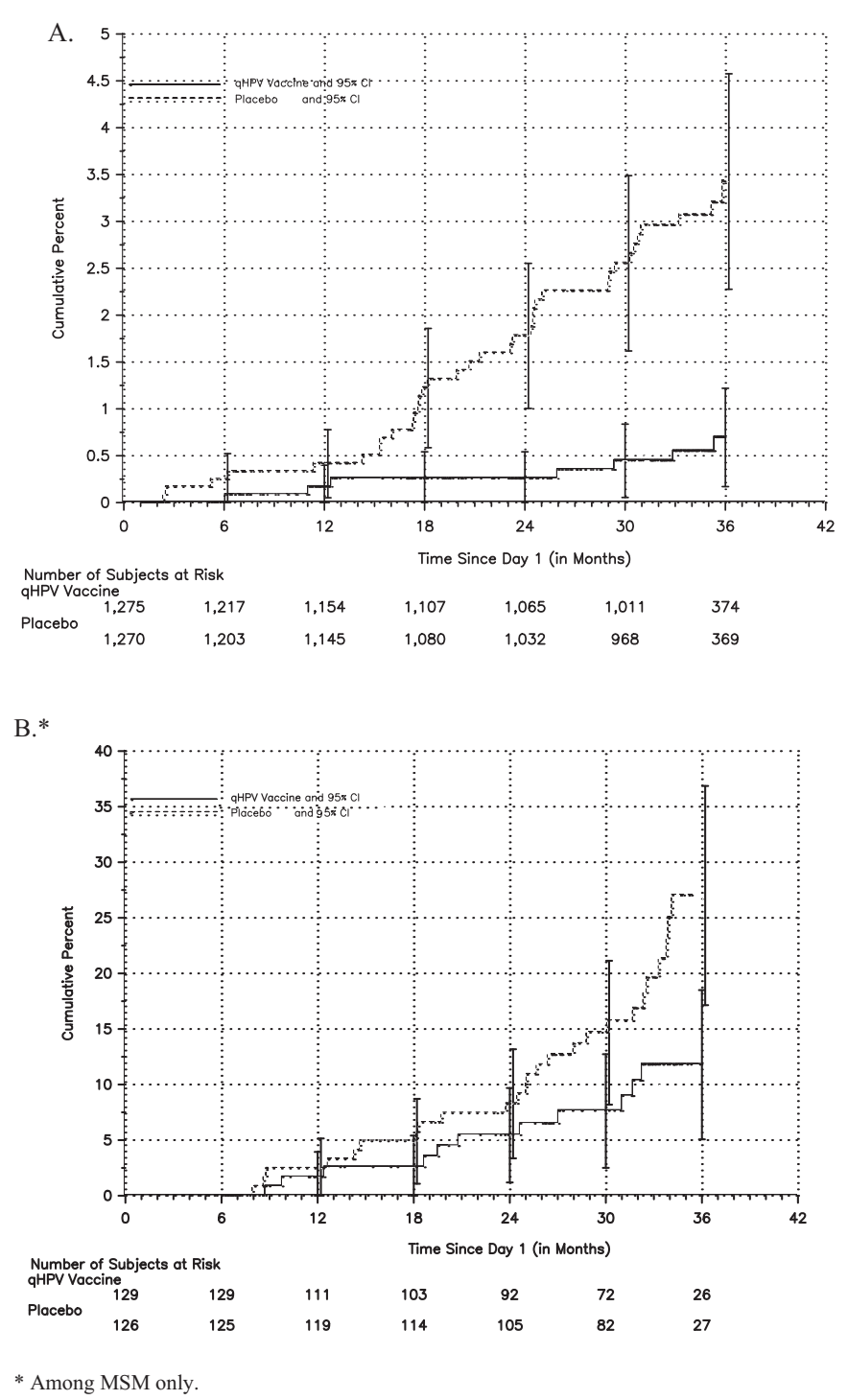

Fig. 2. Analysis of time to (A) EGL and (B) AIN or worse regardless of HPV detection among men in the naïve population.

intra-anal disease (MSM only). No efficacy against AIN related to the 10 non-vaccine HPV types was identified in the HPV naïve population ( 5 vaccine cases and 4 placebo cases). When AIN regardless of HPV detection was considered for the HPV-naïve population, a total of 12 cases in the vaccine group and 28 in the placebo group were identified for an efficacy of $54.9 \%$ (95\% CI: 8.4, 79.1). In the ITT population, the efficacy against AIN related to the 10 non-vaccine HPV types tested was $11.8 \%(95 \% \mathrm{CI}$ : $-39.3,44.4)$ with 38 cases in the vaccine group and 44 cases in the placebo group; these data were also not statistically significant. There were 74 cases of AIN regardless of HPV detection in the qHPV group and 103 in the placebo group for a vaccine efficacy of $25.7 \%$ (95\% CI: -1.1, 45.6).

Statistically significant efficacy was achieved against AIN 1 lesions in the HPV 6/11/16/18 group and the 'regardless of HPV detection' group (due primarily to HPV 6/11/16/18 efficacy), but not in the ' 10 tested non-vaccine type' group (Table 3 ). There was no statistically significant efficacy for intra-anal condyloma related to either HPV 6/11/16/18 or regardless of HPV detection.

Fig. 2a is an analysis of the time to an EGL in the HPV-naïve population for the vaccine and placebo groups regardless of HPV detection. From 18 months forward, there is significantly increased incidence of disease in the placebo group. In Fig. 2b, the analysis is of 
Table 1

Analysis of efficacy against EGL in men.

\begin{tabular}{|c|c|c|c|c|c|c|c|c|c|c|}
\hline \multirow[t]{2}{*}{ Endpoint } & \multicolumn{4}{|c|}{ qHPV vaccine $(N=2025)$} & \multicolumn{4}{|c|}{ Placebo $(N=2030)$} & \multirow{2}{*}{$\begin{array}{l}\text { Observed } \\
\text { efficacy (\%) }\end{array}$} & \multirow[t]{2}{*}{$95 \% \mathrm{CI}$} \\
\hline & $n$ & Cases & PYR & Rate & $n$ & Cases & PYR & Rate & & \\
\hline \multicolumn{11}{|l|}{ HPV-naïve population } \\
\hline EGL & 1275 & 7 & 3358.7 & 0.2 & 1270 & 37 & 3283.2 & 1.1 & 81.5 & $(58.0,93.0)$ \\
\hline HPV 6/11/16/18-related EGL & 1275 & 3 & 3360.3 & 0.1 & 1270 & 32 & 3289.0 & 1.0 & 90.8 & $(70.7,98.2)$ \\
\hline HPV 6-related EGL & 1275 & 3 & 3360.3 & 0.1 & 1270 & 21 & 3299.1 & 0.6 & 86.0 & $(53.0,97.3)$ \\
\hline HPV 11-related EGL & 1275 & 1 & 3363.2 & 0.0 & 1270 & 11 & 3310.2 & 0.3 & 91.1 & $(38.4,99.8)$ \\
\hline HPV 16-related EGL & 1275 & 0 & 3364.8 & 0.0 & 1270 & 3 & 3319.7 & 0.1 & 100 & $(-138.7,100)$ \\
\hline HPV 18-related EGL & 1275 & 0 & 3364.8 & 0.0 & 1270 & 0 & 3322.4 & 0.0 & NA & NA \\
\hline EGL related to any of 10 additional HPV types & 1275 & 2 & 3363.2 & 0.1 & 1270 & 6 & 3317.4 & 0.2 & 67.1 & $(-83.9,96.8)$ \\
\hline HPV 31-related EGL & 1275 & 0 & 3364.8 & 0.0 & 1270 & 3 & 3319.2 & 0.1 & 100 & $(-138.7,100)$ \\
\hline HPV 33-related EGL & 1275 & 0 & 3364.8 & 0.0 & 1270 & 1 & 3321.3 & 0.0 & 100 & $(-3749.6,100)$ \\
\hline HPV 35-related EGL & 1275 & 1 & 3363.2 & 0.0 & 1270 & 0 & 3322.4 & 0.0 & NA & NA \\
\hline HPV 39-related EGL & 1275 & 0 & 3364.8 & 0.0 & 1270 & 1 & 3321.9 & 0.0 & 100 & $(-3750.3,100)$ \\
\hline HPV 45-related EGL & 1275 & 0 & 3364.8 & 0.0 & 1270 & 1 & 3320.7 & 0.0 & 100 & $(-3748.9,100)$ \\
\hline HPV 51-related EGL & 1275 & 0 & 3364.8 & 0.0 & 1270 & 0 & 3322.4 & 0.0 & NA & NA \\
\hline HPV 52-related EGL & 1275 & 1 & 3364.8 & 0.0 & 1270 & 2 & 3320.7 & 0.1 & 50.7 & $(-847.9,99.2)$ \\
\hline HPV 56-related EGL & 1275 & 0 & 3364.8 & 0.0 & 1270 & 0 & 3322.4 & 0.0 & NA & NA \\
\hline HPV 58-related EGL & 1275 & 0 & 3364.8 & 0.0 & 1270 & 0 & 3322.4 & 0.0 & NA & NA \\
\hline HPV 59-related EGL & 1275 & 0 & 3364.8 & 0.0 & 1270 & 1 & 3320.9 & 0.0 & 100 & $(-3749.1,100)$ \\
\hline EGL not related to any of 14 tested HPV types & 1275 & 3 & 3349.2 & 0.1 & 1270 & 5 & 3266.5 & 0.2 & 41.5 & $(-200.8,90.9)$ \\
\hline \multicolumn{11}{|l|}{ Intention-to-treat population } \\
\hline EGL & 1943 & 38 & 4971.1 & 0.8 & 1937 & 92 & 4894.5 & 1.9 & 59.3 & $(40.0,72.9)$ \\
\hline HPV 6/11/16/18-related EGL & 1943 & 27 & 4987.0 & 0.5 & 1937 & 80 & 4914.2 & 1.6 & 66.7 & $(48.0,79.3)$ \\
\hline HPV 6-related EGL & 1943 & 21 & 4998.3 & 0.4 & 1937 & 52 & 4943.2 & 1.1 & 60.1 & $(32.5,77.1)$ \\
\hline HPV 11-related EGL & 1943 & 6 & 5029.2 & 0.1 & 1937 & 26 & 4978.9 & 0.5 & 77.2 & $(43.2,92.3)$ \\
\hline HPV 16-related EGL & 1943 & 3 & 5029.6 & 0.1 & 1937 & 11 & 4998.9 & 0.2 & 72.9 & $(-2.6,95.1)$ \\
\hline HPV 18-related EGL & 1943 & 2 & 5035.7 & 0.0 & 1937 & 3 & 5008.5 & 0.1 & 33.7 & $(-478.8,94.5)$ \\
\hline EGL related to any of 10 additional HPV types & 1943 & 9 & 5020.4 & 0.2 & 1937 & 18 & 4989.5 & 0.4 & 50.3 & $(-16.5,80.3)$ \\
\hline HPV 31-related EGL & 1943 & 0 & 5037.8 & 0.0 & 1937 & 5 & 5004.1 & 0.1 & 100 & $(-8.4,100)$ \\
\hline HPV 33-related EGL & 1943 & 1 & 5037.8 & 0.0 & 1937 & 3 & 5007.6 & 0.1 & 66.9 & $(-312.7,99.4)$ \\
\hline HPV 35-related EGL & 1943 & 1 & 5036.2 & 0.0 & 1937 & 0 & 5011.1 & 0.0 & NA & NA \\
\hline HPV 39-related EGL & 1943 & 0 & 5037.8 & 0.0 & 1937 & 1 & 5010.6 & 0.0 & 100 & $(-3778.9,100)$ \\
\hline HPV 45-related EGL & 1943 & 0 & 5037.8 & 0.0 & 1937 & 1 & 5009.4 & 0.0 & 100 & $(-3778.0,100)$ \\
\hline HPV 51-related EGL & 1943 & 1 & 5035.7 & 0.0 & 1937 & 4 & 5008.3 & 0.1 & 75.1 & $(-151.3,99.5)$ \\
\hline HPV 52-related EGL & 1943 & 3 & 5031.3 & 0.1 & 1937 & 4 & 5003.0 & 0.1 & 25.4 & $(-340.8,89.1)$ \\
\hline HPV 56-related EGL & 1943 & 2 & 5033.0 & 0.0 & 1937 & 1 & 5010.7 & 0.0 & -99.1 & $(-11647.1,89.6)$ \\
\hline HPV 58-related EGL & 1943 & 0 & 5037.8 & 0.0 & 1937 & 3 & 5005.4 & 0.1 & 100 & $(-140.4,100)$ \\
\hline HPV 59-related EGL & 1943 & 2 & 5035.2 & 0.0 & 1937 & 1 & 5009.5 & 0.0 & -99.0 & $(-11639.4,89.6)$ \\
\hline EGL not related to any of 14 tested HPV types & 1943 & 8 & 4875.4 & 0.2 & 1937 & 12 & 4792.0 & 0.3 & 34.5 & $(-74.3,76.8)$ \\
\hline
\end{tabular}

Participants are counted once in each applicable endpoint category. A participant may appear in more than one category.

$N$, number of participants randomized to the respective vaccination group who received at least 1 injection.

$n$, number of participants who have at least one follow-up visit after day 1 .

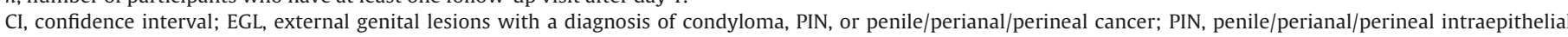
neoplasia; PYR, person years at risk; rate, incidence rate per 100 person years at risk.

time to an intra-anal lesion in the HPV-naïve population for the vaccine and placebo groups regardless of HPV detection. Although the curves move toward greater separation, a statistically significant difference between the vaccine and placebo groups is not achieved by study end at 36 months.

\section{Discussion}

Previous reports have demonstrated qHPV vaccine efficacy against both HPV 6/11/16/18-related EGLs (90.4\% in both HM and MSM) [13] and intra-anal disease (77.5\% in MSM only) in the perprotocol efficacy analyses [20]. This report examines the efficacy against disease caused by 10 additional non-vaccine HPV types, as well as efficacy regardless of whether or not HPV was detected in an EGL or AIN lesion. The presented analyses in the ITT population, as well as the more selective HPV-naïve population, continue to confirm previous publications on the efficacy of the vaccine against AIN and EGLs in men.

While efficacy against EGLs regardless of HPV detection was retained, efficacy was 9.3\% lower at external genital sites and 34.7\% lower at the intra-anal site when compared with efficacy against HPV 6/11/16/18. The larger drop in efficacy at the intra-anal site may be due to the fact that a larger proportion of anal disease is caused by the additional $10 \mathrm{HPV}$ types considered. In the face of little to no cross-protection, this would decrease the observed efficacy at the intra-anal site. In comparison, the majority ( $90 \%)$ of external genital lesions are caused by HPV types 6 and 11. Lack of cross-protection against the additional 10 HPV types considered would have minimal impact on vaccine efficacy when considering protection regardless of HPV detection compared to 4 types. It is important to consider that a larger study would be necessary to make definitive conclusions concerning these assertions.

The greater drop in efficacy for intra-anal disease (with respect to EGLs) may also reflect the fact that the additional 10 HPV types tested were oncogenic types (with respect to cervical cancer) and were more likely to cause moderate to high-grade dysplasia both on the penis and anus than low-risk HPV types. Most EGLs were condyloma that are most often related to HPV 6 and 11, so testing for the additional 10 types on the penis where only 2 cases of PIN2 or 3 were identified would be expected to have less impact. Within the anal canal, however, 25 cases of non-condylomatous lesions were found and non-vaccine HPV types played a larger role. As expected, vaccine efficacy for preventing intra-anal condyloma in the HPVnaïve population dropped from $100 \%$ to $82.3 \%$ in the regardless of 
HPV detection group because most lesions were related to HPV 6 and/or 11, but neither value reached significance, likely due to small sample size.

Efficacy against disease endpoints caused by the 10 non-vaccine types was not demonstrated; however, this conclusion is limited by the study size and person follow-up time. In addition, this study was not powered for this endpoint. The larger female qHPV vaccine clinical trial population was also underpowered for the demonstration of cross-protective efficacy against genital disease related to the same 14 tested individual HPV types [11,12].

Although Fig. 2a demonstrates significant reduction in EGLs regardless of HPV detection in the vaccine group when compared with the placebo group after 18 months, this was not seen for the intra-anal disease (Fig. 2b). However, the short follow-up time in this trial may have allowed the establishment of a protective effect against condyloma and not intranal disease because of the longer time required to develop AIN as opposed to a shorter time for condyloma. The fact that the curves continue to separate with time and trend toward a significant difference is encouraging, although a large part of the combined efficacy in the regardless of HPV detection group is due to the efficacy seen against HPV 6/11/16/18. It is clearly possible that with longer follow-up we might have identified a significant reduction in intra-anal disease in the vaccine group that may require several years to develop from time of infection. It is also possible that this could be related to some degree of cross-protection that was not demonstrated because of small sample size and limited follow-up. The results also likely reflect the fact that there is a greater likelihood for disease end-points within the anal canal to be caused by the additional 10 tested types.

Importantly, the data presented do not support the idea that vaccination with qHPV vaccine will increase the likelihood of disease caused by non-vaccine types in the short-term. While the sample size was small and the mean follow-up approximately 2.5 years, we did not see increased incidence of disease related to the 10 additional HPV types tested or non-HPV detected types for any disease endpoint in either the ITT and HPV-naïve populations in qHPV vaccine participants.

There are several important limitations to this study. The participants enrolled were young with limited numbers of sexual partners. Therefore, the results may not be representative of the population as a whole. The MSM substudy also had a small sample size and the overall study had a relatively short median follow-up of $<3$ years. It is possible that we would have achieved statistical significance for multiple endpoints where the trend toward efficacy is apparent had we enrolled more participants.

Table 2

Analysis of efficacy against AIN and anal cancer in MSM.

\begin{tabular}{|c|c|c|c|c|c|c|c|c|c|c|}
\hline \multirow[t]{2}{*}{ Endpoint } & \multicolumn{4}{|c|}{ qHPV vaccine $(N=299)$} & \multicolumn{4}{|c|}{ Placebo $(N=299)$} & \multirow{2}{*}{$\begin{array}{l}\text { Observed } \\
\text { efficacy (\%) }\end{array}$} & \multirow[t]{2}{*}{$95 \% \mathrm{CI}$} \\
\hline & $n$ & Cases & PYR & Rate & $n$ & Cases & PYR & Rate & & \\
\hline \multicolumn{11}{|l|}{ HPV-naïve population } \\
\hline AIN and anal cancer & 129 & 12 & 299.4 & 4.0 & 126 & 28 & 315.2 & 8.9 & 54.9 & $(8.4,79.1)$ \\
\hline HPV 6/11/16/18-related AIN and anal cancer & 129 & 2 & 305.4 & 0.7 & 126 & 20 & 317.2 & 6.3 & 89.6 & $(57.2,98.8)$ \\
\hline HPV 6-related AIN and anal cancer & 129 & 1 & 306.6 & 0.3 & 126 & 9 & 325.6 & 2.8 & 88.2 & $(14.8,99.7)$ \\
\hline HPV 11-related AIN and anal cancer & 129 & 0 & 308.0 & 0.0 & 126 & 8 & 321.3 & 2.5 & 100 & $(38.9,100)$ \\
\hline HPV 16-related AIN and anal cancer & 129 & 1 & 306.8 & 0.3 & 126 & 3 & 328.3 & 0.9 & 64.3 & $(-344.3,99.3)$ \\
\hline HPV 18-related AIN and anal cancer & 129 & 0 & 308.0 & 0.0 & 126 & 3 & 326.5 & 0.9 & 100 & $(-156.5,100)$ \\
\hline AIN and anal cancer related to any of 10 additional HPV types & 129 & 5 & 304.7 & 1.6 & 126 & 4 & 329.4 & 1.2 & -35.1 & $(-581.0,70.9)$ \\
\hline HPV 31-related AIN and anal cancer & 129 & 0 & 308.0 & 0.0 & 126 & 1 & 329.5 & 0.3 & 100 & $(-4072.8,100)$ \\
\hline HPV 33-related AIN and anal cancer & 129 & 0 & 308.0 & 0.0 & 126 & 0 & 329.5 & 0.0 & NA & NA \\
\hline HPV 35-related AIN and anal cancer & 129 & 0 & 308.0 & 0.0 & 126 & 0 & 329.5 & 0.0 & NA & NA \\
\hline HPV 39-related AIN and anal cancer & 129 & 0 & 308.0 & 0.0 & 126 & 1 & 329.5 & 0.3 & 100 & $(-4072.8,100)$ \\
\hline HPV 45-related AIN and anal cancer & 129 & 2 & 305.4 & 0.7 & 126 & 0 & 329.5 & 0.0 & NA & NA \\
\hline HPV 51-related AIN and anal cancer & 129 & 2 & 308.0 & 0.6 & 126 & 1 & 329.4 & 0.3 & -113.9 & $(-12517.6,88.9)$ \\
\hline HPV 52-related AIN and anal cancer & 129 & 0 & 308.0 & 0.0 & 126 & 1 & 329.5 & 0.3 & 100 & $(-4072.8,100)$ \\
\hline HPV 56-related AIN and anal cancer & 129 & 0 & 308.0 & 0.0 & 126 & 0 & 329.5 & 0.0 & NA & NA \\
\hline HPV 58-related AIN and anal cancer & 129 & 0 & 308.0 & 0.0 & 126 & 0 & 329.5 & 0.0 & NA & NA \\
\hline HPV 59-related AIN and anal cancer & 129 & 1 & 307.3 & 0.3 & 126 & 0 & 329.5 & 0.0 & NA & NA \\
\hline AIN and anal cancer not related to any of 14 tested HPV types & 129 & 7 & 297.9 & 2.3 & 126 & 6 & 308.2 & 1.9 & -20.7 & $(-334.8,65.3)$ \\
\hline \multicolumn{11}{|l|}{ Intention-to-treat population } \\
\hline AIN and anal cancer & 275 & 74 & 569.0 & 13.0 & 276 & 103 & 588.4 & 17.5 & 25.7 & $(-1.1,45.6)$ \\
\hline HPV 6/11/16/18-related AIN and anal cancer & 275 & 38 & 607.1 & 6.3 & 276 & 77 & 611.9 & 12.6 & 50.3 & $(25.7,67.2)$ \\
\hline HPV 6-related AIN and anal cancer & 275 & 18 & 644.8 & 2.8 & 276 & 47 & 645.3 & 7.3 & 61.7 & $(32.8,79.1)$ \\
\hline HPV 11-related AIN and anal cancer & 275 & 13 & 651.2 & 2.0 & 276 & 25 & 660.5 & 3.8 & 47.3 & $(-7.1,75.2)$ \\
\hline HPV 16-related AIN and anal cancer & 275 & 8 & 668.7 & 1.2 & 276 & 18 & 678.6 & 2.7 & 54.9 & $(-9.0,83.0)$ \\
\hline HPV 18-related AIN and anal cancer & 275 & 5 & 671.9 & 0.7 & 276 & 11 & 684.5 & 1.6 & 53.7 & $(-44.6,87.4)$ \\
\hline AIN and anal cancer related to any of 10 additional HPV types & 275 & 38 & 635.4 & 6.0 & 276 & 44 & 648.8 & 6.8 & 11.8 & $(-39.3,44.4)$ \\
\hline HPV 31-related AIN and anal cancer & 275 & 7 & 675.1 & 1.0 & 276 & 8 & 687.1 & 1.2 & 11.0 & $(-181.0,72.5)$ \\
\hline HPV 33-related AIN and anal cancer & 275 & 1 & 677.8 & 0.1 & 276 & 2 & 690.9 & 0.3 & 49.0 & $(-879.0,99.1)$ \\
\hline HPV 35-related AIN and anal cancer & 275 & 3 & 675.2 & 0.4 & 276 & 5 & 687.6 & 0.7 & 38.9 & $(-214.1,90.5)$ \\
\hline HPV 39-related AIN and anal cancer & 275 & 6 & 670.8 & 0.9 & 276 & 8 & 689.3 & 1.2 & 22.9 & $(-153.3,78.0)$ \\
\hline HPV 45-related AIN and anal cancer & 275 & 5 & 671.2 & 0.7 & 276 & 7 & 686.4 & 1.0 & 27.0 & $(-167.3,81.7)$ \\
\hline HPV 51-related AIN and anal cancer & 275 & 9 & 674.0 & 1.3 & 276 & 9 & 683.7 & 1.3 & -1.4 & $(-188.4,64.3)$ \\
\hline HPV 52-related AIN and anal cancer & 275 & 2 & 677.1 & 0.3 & 276 & 7 & 688.1 & 1.0 & 71.0 & $(-52.5,97.1)$ \\
\hline HPV 56-related AIN and anal cancer & 275 & 9 & 666.4 & 1.4 & 276 & 5 & 689.2 & 0.7 & -86.1 & $(-607.0,44.0)$ \\
\hline HPV 58-related AIN and anal cancer & 275 & 5 & 672.4 & 0.7 & 276 & 6 & 686.1 & 0.9 & 15.0 & $(-234.4,79.5)$ \\
\hline HPV 59-related AIN and anal cancer & 275 & 11 & 667.9 & 1.6 & 276 & 9 & 687.8 & 1.3 & -25.9 & $(-243.6,52.6)$ \\
\hline AIN and anal cancer not related to any of 14 tested HPV types & 275 & 15 & 558.7 & 2.7 & 276 & 16 & 566.0 & 2.8 & 5.0 & $(-105.1,56.3)$ \\
\hline
\end{tabular}

Participants are counted once in each applicable endpoint category. A participant may appear in more than one category.

$N$, number of participants in the MSM substudy randomized to the respective vaccination group who received at least 1 injection.

$n$, number of participants in the MSM substudy who have at least one follow-up visit after day 1.

AIN, anal intraepithelial neoplasia; CI, confidence interval; MSM, men having sex with men; PYR, person years at risk; rate, incidence rate per 100 person years at risk. 
Table 3

Analysis of efficacy against PIN 1 or worse, PIN 2/3 or worse, AIN 1, AIN 2/3 or worse, intra-anal condyloma and genital warts due to any HPV type in HPV naïve men.

\begin{tabular}{|c|c|c|c|c|c|c|c|c|c|c|}
\hline \multirow[t]{2}{*}{ Endpoint } & \multicolumn{4}{|c|}{ qHPV vaccine $(N=2025)$} & \multicolumn{4}{|c|}{ Placebo $(N=2030)$} & \multirow{2}{*}{$\begin{array}{l}\text { Observed } \\
\text { efficacy (\%) }\end{array}$} & \multirow[t]{2}{*}{$95 \% \mathrm{CI}$} \\
\hline & $n$ & Cases & PYR & Rate & $n$ & Cases & PYR & Rate & & \\
\hline PIN 1 or worse ${ }^{a}$ & 1275 & 2 & 3364.2 & 0.1 & 1270 & 4 & 3320.3 & 0.1 & 50.7 & $(-244.3,95.5)$ \\
\hline Related to HPV 6/11/16/18 & 1275 & 0 & 3364.8 & 0.0 & 1270 & 3 & 3320.7 & 0.1 & 100 & $(-138.8,100)$ \\
\hline Related to any of 10 additional HPV types & 1275 & 0 & 3364.8 & 0.0 & 1270 & 1 & 3322.2 & 0.0 & 100 & $(-3750.7,100)$ \\
\hline Not related to any of 14 tested HPV types & 1275 & 2 & 3350.2 & 0.1 & 1270 & 1 & 3271.9 & 0.0 & -95.3 & $(-11423.8,89.8)$ \\
\hline PIN 2/3 or worse ${ }^{b}$ & 1275 & 0 & 3364.8 & 0.0 & 1270 & 2 & 3320.7 & 0.1 & 100 & $(-425.5,100)$ \\
\hline Related to HPV 6/11/16/18 & 1275 & 0 & 3364.8 & 0.0 & 1270 & 2 & 3320.7 & 0.1 & 100 & $(-425.5,100)$ \\
\hline Related to any of 10 additional HPV types & 1275 & 0 & 3364.8 & 0.0 & 1270 & 1 & 3322.2 & 0.0 & 100 & $(-3750.7,100)$ \\
\hline Not related to any of 14 tested HPV types & 1275 & 0 & 3350.8 & 0.0 & 1270 & 0 & 3272.4 & 0.0 & NA & NA \\
\hline $\operatorname{AIN} 1^{c}$ & 129 & 6 & 305.3 & 2.0 & 126 & 19 & 317.4 & 6.0 & 67.2 & $(14.5,89.3)$ \\
\hline Related to HPV 6/11/16/18 & 129 & 1 & 306.8 & 0.3 & 126 & 15 & 318.9 & 4.7 & 93.1 & $(55.0,99.8)$ \\
\hline Related to any of 10 additional HPV types & 129 & 2 & 306.8 & 0.7 & 126 & 2 & 329.4 & 0.6 & -7.4 & $(-1381.0,92.2)$ \\
\hline Not related to any of 14 tested HPV types & 129 & 4 & 301.6 & 1.3 & 126 & 3 & 310.2 & 1.0 & -37.1 & $(-836.2,76.8)$ \\
\hline Non-acuminate AIN $1^{\mathrm{c}}$ & 129 & 5 & 306.6 & 1.6 & 126 & 15 & 321.1 & 4.7 & 65.1 & $(-1.0,90.1)$ \\
\hline Related to HPV 6/11/16/18 & 129 & 1 & 306.8 & 0.3 & 126 & 12 & 321.9 & 3.7 & 91.3 & $(40.9,99.8)$ \\
\hline Related to any of 10 additional HPV types & 129 & 2 & 306.8 & 0.7 & 126 & 2 & 329.4 & 0.6 & -7.4 & $(-1381.0,92.2)$ \\
\hline Not related to any of 14 tested HPV types & 129 & 3 & 302.9 & 1.0 & 126 & 2 & 310.9 & 0.6 & -54.0 & $(-1743.4,82.4)$ \\
\hline Condyloma acuminatum & 1275 & 5 & 3359.3 & 0.1 & 1270 & 33 & 3285.3 & 1.0 & 85.2 & $(61.8,95.5)$ \\
\hline Related to HPV 6/11/16/18 & 1275 & 3 & 3360.3 & 0.1 & 1270 & 29 & 3290.7 & 0.9 & 89.9 & $(67.3,98.0)$ \\
\hline Related to any of 10 additional HPV types & 1275 & 2 & 3363.2 & 0.1 & 1270 & 5 & 3317.6 & 0.2 & 60.5 & $(-141.0,96.2)$ \\
\hline Not related to any of 14 tested HPV types & 1275 & 1 & 3349.8 & 0.0 & 1270 & 4 & 3267.0 & 0.1 & 75.6 & $(-146.4,99.5)$ \\
\hline Intra-anal condyloma acuminatum $^{\mathrm{c}}$ & 129 & 1 & 306.6 & 0.3 & 126 & 6 & 325.8 & 1.8 & 82.3 & $(-46.0,99.6)$ \\
\hline Related to HPV 6/11/16/18 & 129 & 0 & 308.0 & 0.0 & 126 & 5 & 326.6 & 1.5 & 100 & $(-15.7,100)$ \\
\hline Related to any of 10 additional HPV types & 129 & 0 & 308.0 & 0.0 & 126 & 0 & 329.5 & 0.0 & NA & NA \\
\hline Not related to any of 14 tested HPV types & 129 & 1 & 301.7 & 0.3 & 126 & 1 & 309.4 & 0.3 & -2.6 & $(-7950.0,98.7)$ \\
\hline
\end{tabular}

Participants are counted once in each applicable endpoint category. A participant may appear in more than one category.

$N$, number of participants randomized to the respective vaccination group who received at least 1 injection.

$n$, number of participants who have at least one follow-up visit after day 1.

CI, confidence interval; PIN, penile/perianal/perineal intraepithelial neoplasia; AIN, anal intraepithelial neoplasia.

a PIN 1 or worse is defined as external genital lesions with a diagnosis of PIN 1, PIN 2/3, or penile/perianal/perineal cancer.

b PIN 2/3 or worse defined as PIN 2/3 or penile/perianal/perineal cancer.

c MSM participants only.

In conclusion, the data presented suggest that vaccinating males against HPV 6, 11, 16 and 18 protects them from most HPVrelated disease; although significant efficacy against non-vaccine HPV types was not seen. In addition, the data do not support the theory that vaccination with qHPV vaccine will increase the likelihood of disease caused by non-vaccine types, however, additional studies (including population-based surveillance and effectiveness studies) with longer follow-up time would be required to investigate this further.

\section{Financial support}

Merck \& Co, Inc.

\section{Conflicts of interest statement}

EM, AG and JP have received research grants from Merck, either personally or through their institution. DGF and AG have received research grants from GlaxoSmithKline. AG, SG, DGF, EV, $\mathrm{RJH}, \mathrm{JP}$ and EM have received honoraria from Merck for speaking engagements or board membership either personally or through their institutions. SG, DGF, JP and EM have received travel reimbursement from Merck related to scientific meetings. CA has an approved, filed or pending patent related to participant matter discussed in this manuscript. EG, RMH, DG, JBM and SV are employees of Merck and may own Merck stock and/or stock options.

\section{Contributors}

The trial was designed by the sponsor (Merck \& Co, Inc.) in collaboration with external investigators (AG, JP, SG) and an external data and safety monitoring board. The sponsor collated data, monitored the conduct of the trial (EG, DG, RMH), performed statistical analyses (KLL, JBM) and coordinated manuscript writing with all authors (SV). Authors were actively involved in the collection, analysis and interpretation of the data, creation and revision of the manuscript for intellectual content, and approval of the final manuscript. The first draft was written by SG and SV, with contributions from all other authors. All authors met the ICMJE guidelines for authorship, had access to data (with confidentiality agreements) and took part in the decision on where to submit the manuscript for publication.

\section{References}

[1] World Health Organization. Human papillomavirus infection and cervical cancer. World Health Organization; 2004, available from http://www.who.int/vaccine_research/diseases/hpv/en/ [accessed 05.07.07].

[2] Giuliano AR, Lee JH, Fulp W, Villa LL, Lazcano E, Papenfuss MR, et al. Incidence and clearance of genital human papillomavirus infection in men (HIM): a cohort study. Lancet 2011;377:932-40.

[3] Wiley DJ, Douglas J, Beutner K, Cox T, Fife K, Moscicki AB, et al. External genital warts: diagnosis, treatment and prevention. Clinical Infectious Diseases 2002;35:S210-24

[4] Palefsky JM. Human papillomavirus-related disease in men: not just a women's issue. Journal of Adolescent Health 2010;46:S12-9. 
[5] Giuliano AR, Anic G, Nyitray AG. Epidemiology and pathology of HPV disease in males. Gynecologic Oncology 2010;117:S15-9.

[6] Chan PK, Ho WC, Yu MY, Pong WM, Chan AC, Chan AK, et al. Distribution of human papillomavirus types in cervical cancers in Hong Kong: current situation and changes over the last decades. International Journal of Cancer 2009; $125: 1671-7$.

[7] Aubin F, Pretet JL, Jacquard AC, Saunier M, Carcopino X, Jaroud F, et al. Human papillomavirus genotype distribution in external acuminata condylomata: a Large French National Study (EDiTH IV). Clinical Infectious Diseases 2008;47:610-5.

[8] Anic GM, Lee JH, Stockwell H, Rollison DE, Wu Y, Papenfuss MR, et al. Incidence and human papillomavirus (HPV) type distribution of genital warts in a multinational cohort of men: the HPV in men study. Journal of Infectious Diseases 2011;204:1886-92.

[9] Muñoz N, Castellsague X, de Gonzalez AB, Gissman L. HPV in the etiology of human cancer. Vaccine 2006;24:S1-10.

[10] Bouvard V, Baan R, Straif K, Grosse Y, Secretan B, El Ghissassi F, et al. A review of human carcinogens-part B: biological agents. Lancet Oncology 2009; 10:321-2.

[11] Wheeler C, Kjaer SK, Sigurdsson K, Iversen O, Hernandez-Avila M, Perez G, et al. The impact of quadrivalent HPV (type 6/11/16/18) L1 VLP vaccine on infection and disease due to oncogenic non-vaccine HPV types in sexually active women aged 16-26. Journal of Infectious Diseases 2009;199:936-44.

[12] Brown DR, Kjaer SK, Sigurdsson K, Iversen O-E, Hernandez-Avila M, Wheeler CM, et al. The impact of quadrivalent HPV (type 6/11/16/18) L1 VLP vaccine on infection and disease due to oncogenic non-vaccine HPV types in generally HPV naive women aged 16-26. Journal of Infectious Diseases 2009;199:926-35.

[13] Giuliano AR, Palefsky JM, Goldstone S, Moreira Jr ED, Penny ME, Aranda C, et al. Efficacy of quadrivalent HPV vaccine against HPV Infection and disease in males. New England Journal of Medicine 2011;364:401-11.

[14] Henry MR. The Bethesda system 2001: an update of new terminology for gynecologic cytology. Clinics in Laboratory Medicine 2003;23:585-603.

[15] Garland SM, Hernandez-Avila M, Wheeler CM, Perez G, Harper DM, Leodolter S, et al. Quadrivalent vaccine against human papillomavirus to prevent anogenital diseases. New England Journal of Medicine 2007;356:1928-43.

[16] The FUTURE II Study Group. Quadrivalent vaccine against human papillomavirus to prevent high-grade cervical lesions. New England Journal of Medicine 2007;356:1915-27.

[17] Weaver BA, Feng Q, Holmes KK, Kiviat N, Lee SK, Meyer C, et al. Evaluation of genital sites and sampling techniques for detection of human papillomavirus DNA in men. Journal of Infectious Diseases 2004;189:677-85.

[18] Vardas E, Giuliano A, Goldstone Palefsky J, Moreira Jr ED, Penny ME, et al. External genital HPV prevalence and associated factors among heterosexual men on five continents. Journal of Infectious Diseases 2010;203:58-65.

[19] Goldstone SE, Palefsky J, Giuliano A, Moreira Jr ED, Aranda C, Jessen H, et al. Prevalence and risk factors for HPV infection in HIV seronegative men who have sex with men. Journal of Infectious Diseases 2010;203:66-74.

[20] Palefsky JM, Giuliano AR, Goldstone S, Moreira ED, Aranda C, Jessen H, et al. HPV vaccine against anal HPV infection and anal intraepithelial neoplasia. New England Journal of Medicine 2011;365:1576-85. 